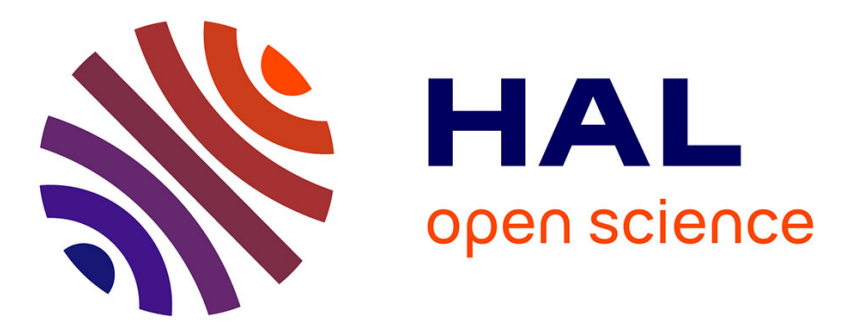

\title{
Impact of prone positioning in infants with Pierre Robin sequence: a polysomnography study
}

\author{
L. Coutier, A. Guyon, P. Reix, P. Franco
}

\section{To cite this version:}

L. Coutier, A. Guyon, P. Reix, P. Franco. Impact of prone positioning in infants with Pierre Robin sequence: a polysomnography study. Sleep Medicine, 2019, 54, pp.257 - 261. 10.1016/j.sleep.2018.10.037 . hal-03486396

\section{HAL Id: hal-03486396 https://hal.science/hal-03486396}

Submitted on 20 Dec 2021

HAL is a multi-disciplinary open access archive for the deposit and dissemination of scientific research documents, whether they are published or not. The documents may come from teaching and research institutions in France or abroad, or from public or private research centers.
L'archive ouverte pluridisciplinaire HAL, est destinée au dépôt et à la diffusion de documents scientifiques de niveau recherche, publiés ou non, émanant des établissements d'enseignement et de recherche français ou étrangers, des laboratoires publics ou privés.

\section{다)(1) $(5$}

Distributed under a Creative Commons Attribution - NonCommerciall 4.0 International 


\title{
Impact of prone positioning in infants with Pierre Robin sequence: a polysomnography study
}

\author{
L. Coutier ${ }^{\mathrm{a}, \mathrm{b}}, \mathrm{MD}, \mathrm{A}$. Guyon $^{\mathrm{b}}, \mathrm{PhD}, \mathrm{P} . \mathrm{Reix}^{\mathrm{a}, \mathrm{c}}, \mathrm{MD}, \mathrm{PhD}, \mathrm{P}$. Franco ${ }^{\mathrm{b}}, \mathrm{MD}, \mathrm{PhD}$
}

Affiliations: ${ }^{a}$ Service de pneumologie infantile, allergologie et centre de référence en mucoviscidose, Hôpital Femme Mère Enfant, Hospices Civils de Lyon, Bron, France; ${ }^{\mathrm{b}}$ Service d'épileptologie clinique, des troubles du sommeil et de neurologie fonctionnelle de l'enfant, Hôpital Femme Mère Enfant, Hospices Civils de Lyon, Bron, U1028, CNRL, Université Lyon 1, France; 'UMR 5558 (EMET). CNRS, LBBE, Université de Lyon, Villeurbanne, France

Address for correspondence: Laurianne Coutier, Service de pneumologie infantile, allergologie et centre de référence en mucoviscidose, Hôpital Femme Mère Enfant, 59; boulevard Pinel, 69500 Bron, France, [laurianne.coutier@chu-lyon.fr], +33(0)472129437

Funding Source: No external funding for this manuscript.

Financial Disclosure: The authors have indicated they have no financial relationships relevant to this article to disclose.

Conflict of Interest: The authors have indicated they have no potential conflicts of interest to disclose.

Clinical Trial Registration: Not applicable

Abbreviations: arousal index (AI), central apnea index (CAI), interquartile range (IQR), mixed apnea index (MAI), non-invasive ventilation (NIV), nasopharyngeal tube (NPT), nonrapid eye movement stage 1 (NREM1), non-rapid eye movement stage 2 (NREM2), obstructive apnea-hypopnea index (OAHI), obstructive apnea index (OAI), oxygen desaturation index over 3\% (OD3\%), oxygen desaturation index over 4\% (OD4\%), obstructive hypopnea index (OHI), obstructive sleep apnea (OSA), obstructive sleep apnea syndrome (OSAS), prone positioning (PP), Pierre Robin sequence (PRS), polygraphy (PG), polysomnography (PSG), transcutaneous carbon dioxide pressure $(\mathrm{P}(\mathrm{tcCO}(2)))$, respiratory arousals index (RAI), rapid eye movement stage (REM), supine position (SP), total sleep time (TST)

\section{Contributors' Statement Page}

Dr. Coutier conceptualized and designed the study, designed the data collection instruments, collected data, carried out the initial analyses, drafted the initial manuscript, and reviewed and revised the manuscript.

Dr. Guyon designed the study, collected data, carried out the initial analyses, reviewed and revised the manuscript. 
Prof Reix conceptualized the study and critically reviewed the manuscript for important intellectual content.

Prof Franco conceptualized and designed the study, supervised data collection, and critically reviewed the manuscript for important intellectual content.

All authors approved the final manuscript as submitted and agree to be accountable for all aspects of the work. 


\begin{abstract}
Objective/ background Obstructive sleep apnea syndrome (OSA) is frequent in Pierre Robin sequence (PRS) infants. Prone positioning (PP) is commonly recommended but has never been studied by polysomnography (PSG). This study aimed to evaluate the impact of the PP on sleep and breathing outcomes measured by PSG.

Patients / Methods Retrospective study conducted between 2015-2017 in a tertiary hospital. A PSG with pulse oximetry and transcutaneous carbon dioxide was performed in PRS infants in the supine position (SP) and the PP. Sleep and breathing outcome measures were compared between SP and PP.

Results Among the 18 PRS (mean \pm SD age: $44 \pm 26$ days at evaluation), 11 had clinical manifestations of OSA. All had severe OSA diagnosed on PSG. In the PP, infants had a significantly higher sleep efficiency (median [IQR]: 83\% [69-90]) than in the SP (70\% [5577], $\mathrm{p}=0.04)$. During REM, there was a trend towards lower OAHI in the PP $(50 / \mathrm{h}$ [28-82] versus $61 / \mathrm{h}$ [40-103], $\mathrm{p}=0.05)$. For 13 , the $\mathrm{PP}$ was the best sleep position (72\%), and for four the SP was the best sleep position $(22 \% ; \mathrm{p}<0.01)$. The PP was sufficient alone to decrease OSA index $<10$ events/hour in 3 infants.

Conclusion Positioning infants in the PP led to an improvement of sleep quality and an incomplete correction of OSAS in the vast majority of PRS infants. A nocturnal sleep recording seems to be indicated systematically in the early evaluation of these young patients to choose the best therapeutic option for OSAS.
\end{abstract}

Keywords: prone positioning, sleep, polysomnography, pediatric, infants, Pierre Robin sequence 


\section{Introduction}

Prone positioning (PP) is commonly recommended and used in patients with Pierre Robin sequence (PRS) to treat obstructive sleep apnea syndrome (OSAS) ${ }^{1}$. Although, the clinical advantage of PP on breathing outcome measures has been evaluated by a complete exploration such as polysomnography (PSG) ${ }^{1}$, there are no results about the impact of PP on sleep characteristics. The incidence of PRS, defined by the clinical triad of retrognathism, glossoptosis, and cleft palate, is reported to be $1 / 8500$ births in the United Kingdom ${ }^{2}$ and as little as $1 / 10000$ births in France ${ }^{3}$; neonatal clinical manifestations are mainly related to upper airway obstruction and swallowing disorders ${ }^{3}$. No data are available regarding the quality of sleep as measured by PSG in PRS patients while it is the gold standard for OSAS diagnosis ${ }^{4}$, possibly owing to difficulties in accessing this exam ${ }^{1}$. Clinical manifestations in PRS are of variable severity ${ }^{5-7}$. Despite OSAS being one of the most frequent manifestations in $\mathrm{PRS}^{8}$, linked to multifactorial etiologies, a clinical evaluation with specific screening alone may underestimate its severity ${ }^{6,7,9}$. Because OSAS morbidity is associated with impaired cognitive and behavioral development ${ }^{10,11}$, as well as a predisposition to pulmonary hypertension ${ }^{12}$, an early diagnosis should be a priority to establish appropriate management. There are several physiological diagnostic tools to diagnose OSAS, such as PG or PSG, combined with oximetry and transcutaneous carbon dioxide pressure ${ }^{4,13}$, but there is no international consensus as to their use ${ }^{1,6}$. In infants, OSA is classified as mild (apneahypopnea index - AHI $>1-5$ episodes/h), as moderate $(\mathrm{AHI}>5-10$ episodes $/ \mathrm{h})$ or severe $\left(\mathrm{AHI}>10\right.$ episodes/h) ${ }^{14}$. Once confirmed in PRS, OSAS management, without consensus, is based on a combination of non-surgical treatments (PP, non-invasive ventilation - NIV, or nasopharyngeal tube - NPT) and surgical treatments of glossoptosis and cleft palate ${ }^{6,15}$. In current practice, for non-severe OSAS, PP will be used in first-line therapy ${ }^{1,16}$. For severe 
OSAS, PP will be used in first-line therapy, and NIV, NPT or surgery will be used in secondline therapy. For very severe OSAS, NIV, NPT ${ }^{6,15}$ or surgery will be used in first-line therapy. The clinical and physiological benefits of NIV for severe OSAS have been widely studied ${ }^{5,8}$ whereas the benefits of PP have not ${ }^{1}$. The aim of the present study was, therefore, to assess the impact of PP, used in first-line therapy, on both sleep and breathing quality as evaluated by PSG in infants with PRS. 


\section{Material and Methods}

\section{Study design}

\section{Patients}

All infants aged $<8$ months with a PRS diagnosis who were hospitalized in our university hospital, from January 2015 to October 2017, were referred to our center for a complete sleep study by PSG at the sleep unit of the Pediatric University Hospital of Lyon (Hospices Civils de Lyon, France) and were included in this study. Neonatologists, pediatric pulmonologists, ENTs or maxillofacial surgeons who took care of a PRS infant sent the patient to the sleep unit, where PSG was systematically performed, regardless of the presence of clinical OSAS. It is our current practice to perform PSG in patients with PRS. PRS infants were grouped according to the most frequent classification used in the literature ${ }^{1}$ : isolated PRS, bone disease PRS, associated or syndromic PRS. The main clinical characteristics and respiratory support (PP or supine position - SP - for sleeping, NIV, NPT) were collected from medical files. Data were retrospectively analyzed.

\section{Nocturnal PSG}

An overnight PSG was performed in the sleep center. Infants were systematically studied in the SP then in the PP for a minimum of 2 sleep cycles for each condition; if the SP was not tolerated, the infant was excluded from this study. Raw PSG data were analyzed by a single reviewer (L.C.) who was blinded as to sleep position during the overnight sleep study. The nocturnal PSG was performed in the presence of the child's father/mother or guardian, without tilting of the mattress, and using a Morpheus recorder (Micromed, Mogliano Veneto, Italy). Sleep and respiratory events were scored using sleepRT software (OSG, Rumst, Belgium). The PSG recording employed frontal, central, and occipital leads (FP1, FP2, C3, 
$\mathrm{C} 4, \mathrm{O} 1, \mathrm{O} 2, \mathrm{~A} 1$, and $\mathrm{A} 2$ ), video, 2 electrooculograms, 1 chin electromyogram, and 1 electrocardiogram, inductance plethysmography of chest and abdominal respiratory movements, as well as a nasal cannula, oronasal thermistor and saturation values.

\section{Sleep analysis}

The duration of sleep stages (rapid eye movement stage - REM, non-rapid eye movement stage 1 - NREM1 and non-rapid eye movement stage 2 - NREM2,) were scored according to standard guidelines ${ }^{17,18}$. Total sleep time (TST), sleep efficiency (TST/time in bed*100), sleep efficacy (TST/duration of sleep*100), arousal index (AI), and respiratory arousal index (RAI) were calculated.

\section{Respiratory analysis}

Respiratory parameters were defined according to the American Academy of Sleep Medicine 2012 guidelines ${ }^{19}$. Obstructive apnea-hypopnea index (OAHI), obstructive apnea index (OAI), obstructive hypopnea index (OHI), central apnea index (CAI), mixed apnea index (MAI), mean SpO2, time spent with SpO2 values below 90\%, oxygen desaturation index over $3 \%(\mathrm{OD} 3 \%)$, and oxygen desaturation index over 4\% (OD4\%) were collected. Indices of events are expressed as the mean number of events per hour. OAHI was defined as the sum of OAI, OHI, and MAI. OAHI was considered as normal in healthy infants under one event/hour. OSA was classified as a mild (apnea-hypopnea index - AHI > 1-5 episodes/h), as moderate $\left(\mathrm{AHI}>5-10\right.$ episodes/h) or severe $\left(\mathrm{AHI}>10\right.$ episodes/h) ${ }^{14}$. Respiratory analysis was performed for the total duration of sleep then separated according to REM sleep and NREM sleep. Respiratory parameters were analyzed according to the sleep stage and depending on the sleep position. 
Nocturnal carbon dioxide monitoring

Transcutaneous carbon dioxide pressure $\mathrm{P}(\mathrm{tcCO}(2))$ values were obtained over the entire PSG recording period using SenTec system (SenTec Digital Monitor, Therwil, Switzerland). The mean and maximal $\mathrm{P}(\operatorname{tcCO}(2))$, as well as the proportion of time spent with $\mathrm{P}(\operatorname{tcCO}(2))$ over $50 \mathrm{mmHg}$, were calculated. Hypoventilation was scored when the $\mathrm{P}(\mathrm{tcCO}(2))$ was $>50 \mathrm{~mm}$ $\mathrm{Hg}$.

\section{Best sleep position}

Best sleep position (PP or SP) was determined from sleep quality (sleep efficiency criteria) and breathing quality (OAHI criteria). For instance, the PP was the best sleep position when: 1) both sleep and breathing quality were better than in SP,2) the breathing quality was better than in SP and the sleep quality equal in SP, or 3) the sleep quality was better than in SP and the breathing quality equal in SP. If the sleep and breathing quality were equal in PP and SP, the sleep position was considered as equal in PP and SP. When sleep quality was better in PP and breathing quality was better in SP, and the converse, the best sleep position was selected according to breathing quality. Sleep and breathing quality were considered better in PP than in SP when the difference between PP and SP was greater than 5\%; they were considered as equal when the difference between PP and SP was less than 5\%.

\section{Regulatory aspects}

The study protocol was approved by the hospital's ethics committee and the national data protection agency (Commission Nationale de l'informatique et des libertés, number 18-006). 
According to the most recent French law for conducting retrospective clinical research, informed consent was not required; those included were informed of the conduct of the study by an information letter.

\section{Statistical analysis}

Data are presented as the median and interquartile range [IQR]. Sleep and respiratory characteristics were compared between the PP and the SP using the non-parametric test, paired Wilcoxon rank test and the Chi2 test. All analyses were performed using R software (R-project, Rcmdr library, R Development Core Team ${ }^{20}$ ). A $p$ value of less than 0.05 was considered statistically significant. 


\section{Results}

\section{$\underline{\text { Patient characteristics }}$}

PSG recordings were performed in 21 infants with PRS (11 girls, ten boys) aged (median [IQR]) 44 days [27-70]; 3 children were excluded because they did not tolerate sleep in the SP $(\mathrm{OAHI}=15 / \mathrm{h}$ in the PP). In total, PSG data were available for both SP and PP positions for 18 infants. Among these, 12 infants (67\%) presented an isolated PRS and 6 (33\%) an associated or syndromic PRS, (syndromic: Franceschetti $n=2 / 6$, Rubinstein-Taybi $n=1 / 6$, del22q11 n=1/6; associated: $n=2 / 6$ ). The mean \pm SD age at the time of PSG was $44 \pm 26$ days. A total of $12(67 \%)$ infants had respiratory symptoms at the time of the recording (snoring or noisy breathing or apneas or apparent life-threatening events or frequent arousals because of respiratory effort or desaturation or stridor). There were 15 (83\%) infants who did not have respiratory support; before PSG, the PP was used for 14 infants, and the SP was used for 4 infants ( 1 in ambient air and 3 with respiratory support). 2 already had NIV (CPAP settings: 6 $\mathrm{cmH}_{2} \mathrm{O}$ ), and 1 had an NPT. Sucking-swallowing disorders or gastroesophageal reflux was present in $16(89 \%)$ infants, and $11(61 \%)$ had been fed through a nasogastric tube during the days or weeks before PSG was performed and three still had the nasogastric tube during the PSG recording (Table 1).

\section{$\underline{\text { Sleep outcomes measures in the SP and PP }}$}

Median TST was 153 minutes in the SP and 170 minutes in the PP. In the PP, infants had a significantly higher sleep efficiency (median [IQR]: 83\% [69-90]) than in the SP (70\% [5577], $\mathrm{p}=0.04$ ). Sleep efficacy was higher in PP than in the SP, but there was no significant difference $(85 \%$ [74-91] versus $73 \%$ [56-84], $\mathrm{p}=0.11)$. In the PP, there was a trend towards lower RAI (15\% [6-25] versus 19\% [11-28], $\mathrm{p}=0.06)$. The proportion of NREM1 was lower in 
PP than in SP with a statistically significant difference (15\% [11-20] versus 19\% [12-29], $\mathrm{p}=0.03$; Table 2).

\section{$\underline{\text { Respiratory outcome measures in the SP and PP }}$}

In the SP, all infants had a severe OAHI value (> 10/h) among whom $75 \%$ had a very severe OSA (OHAI > 25/h), in the PP 2 infants had a moderate OAHI value (5-10/h; Figure 1). During TST, there was a trend towards lower OAI in the PP (median [IQR]: 9/h [4-34] versus 21/h [9-34], $\mathrm{p}=0.06)$. During REM, there was a trend towards lower OAHI in the PP $(50 / \mathrm{h}$ [28-82] versus 61/h [40-103], $\mathrm{p}=0.05)$. CAI was not significantly different between the two positions. OD4\% was significantly lower in the PP than in the SP (13/h [5-31] versus $22 / \mathrm{h}$ [860], $\mathrm{p}=0.03)$ and there was trend toward lower OD3\% (27/h [11-42] versus 34/h [10-77], $\mathrm{p}=0.10) . \mathrm{P}(\operatorname{tcCO}(2))$ was not significantly different between the two positions (Table 3 ).

\section{The best sleep position according to sleep quality and breathing quality}

For 13 infants the PP was the best sleep position (72\%), and for four infants the SP was the best sleep position (22\%; $\mathrm{p}<0.01)$. The PP and SP were equal for one infant.

Among the 13 infants for whom the PP was the best sleep position, breathing quality was improved for 12/13 (93\%) and sleep quality was improved for 11/13 (84\%) infants. Among the four infants for whom the SP was the best sleep position, breathing quality was improved for 4/4 (100\%) and sleep quality was improved for 1/4 (25\%) infants.

\section{$\underline{\text { Therapeutic option selected after PSG }}$}

The PP was the therapeutic option selected for 3/18 infants, and the SP was the therapeutic option selected for 2/18 infants, 1 in SP before the PSG and 1 in PP before the PSG. 
Respiratory support was needed for 13/18 infants: NIV was initiated for 8/18 infants, the pressure was increased for 2/18 already receiving NIV, and NPT was used for 3/18. 


\section{Discussion}

The present study found that although PP improved breathing quality and sleep quality for two-thirds of infants, it was sufficient to decrease the OSA index below the severe level only for 3 of the 18 patients studied. Interestingly, it was also found that among infants with PRS, a third did not present OSA clinical manifestations although all of them had a severe OSAS $(\mathrm{OAHI}>10 / \mathrm{h})$ as assessed by PSG and three-quarters had a very severe OSA (OHAI > 25/h). This is in accordance with a previous study that has published data on the incidence of OSAS in PRS; Daniel et al. report $75 \%$ of severe OSA in a cohort of 33 PRS infants - the slightly lower incidence may be explained by the age of the patients (mean: 12 months) ${ }^{14}$. It appears from the present study that clinical evaluation alone underestimates both the frequency and the severity of OSAS in PRS. This is also reported by MacLean et al. ${ }^{21}$, and Cielo et al. ${ }^{9}$ who found that sleep disorders, breathing symptoms, and the Brouillette questionnaire correlated poorly with PSG findings for infants with cleft palates. This observation underlines the observation that it is not possible to detect OSAS clinically with sufficient accuracy in infants with PSG.

In the PP, sleep quality was improved for two-thirds of infants compared to the SP, as reflected by the normalization of sleep efficiency and sleep efficacy and the decrease of NREM1. This is the first study to present these outcomes measured by PSG in infants with PRS and confirms the physiological benefits of PP on sleep quality. At the same time, quality of breathing was partially improved for two thirds of infants in the PP compared to the SP with a trend toward a decrease of OAHI, OAI, OD3\%, and a significant decrease of OD4\%. However, despite this improvement, most infants remained above the cut-off level of 10 events/hour that defines severe OSAS. This gain in breathing quality in the PP could explain part of the improvement of sleep quality, as described by Reddy et al. ${ }^{6}$ and Montemitro et al. ${ }^{22}$ but also to the decrease of upper airway obstruction related to glossoptosis and upper 
airway increased collapsibility. However, as mentioned earlier, the improvement in breathing quality in the PP was not sufficient to fully control OSAS; two-thirds of the infants remained with severe OSAS.

An improvement in sleep and breathing quality in the PP was not observed in one third of the patients. This is discrepant with other reports that found a good response to $\mathrm{PP}^{21,22}$. The results presented here indicate that the PP should not be used systematically as the most appropriate sleep position for infants with PRS; it should be evaluated using sleep and respiratory outcome measures, as is already the case for the establishment of NIV in PRS infants ${ }^{5,23}$. Currently, although PSG is considered the gold standard ${ }^{4}$, access to it is still difficult and therefore PG combined with carbon dioxide monitoring with oximetry is commonly used. Additional studies are therefore needed to evaluate PG with respect to PSG for the evaluation of obstructive sleep disordered breathing in patients with PRS.

This study does have certain limitations. It is a retrospective study that included relatively few patients; nevertheless, infants were all systematically assessed in a similar manner in two sleeping positions, prior to their $8^{\text {th }}$ month of life. An additional point is that the statistical analysis did not include a multivariate analysis, which was not appropriate given the number of subject. Furthermore, infants were referred to the sleep unit by either the pediatric or the neonatal intensive care unit, which could be considered as a selection bias favoring the recruitment of the most severe patients. On the other hand, a third of infants included in the study did not present any respiratory signs of OSAS, which is concordant with the frequencies reported elsewhere ${ }^{14}$, and suggests that the sample was representative.

\section{Conclusion}

Positioning infants in the PP led to an improvement of sleep quality but an incomplete correction of OSAS in the large majority of PRS infants. Systematic nocturnal sleep recording 
is indicated in the early evaluation of these young patients in order to objectively assess the best therapeutic option for OSAS, including PP.

This research did not receive any specific grant from funding agencies in the public, commercial, or not-for-profit sectors.

\section{References}

1. Côté A, Fanous A, Almajed A, Lacroix Y. Pierre Robin sequence: review of diagnostic and treatment challenges. Int J Pediatr Otorhinolaryngol. 2015;79(4):451-464. doi:10.1016/j.ijporl.2015.01.035

2. Bush PG, Williams AJ. Incidence of the Robin Anomalad (Pierre Robin syndrome). Br J Plast Surg. 1983;36(4):434-437.

3. Abadie V, Morisseau-Durand M-P, Beyler C, Manach Y, Couly G. Brainstem dysfunction: a possible neuroembryological pathogenesis of isolated Pierre Robin sequence. Eur J Pediatr. 2002;161(5):275-280. doi:10.1007/s00431-002-0936-6

4. Haute Autorité de Santé. Place et conditions de réalisation de la polysomnographie et de la polygraphie respiratoire dans les troubles du sommeil. 2012.

5. Leboulanger N, Picard A, Soupre V, et al. Physiologic and clinical benefits of noninvasive ventilation in infants with Pierre Robin sequence. Pediatrics. 2010;126(5):e1056-e1063. doi:10.1542/peds.2010-0856

6. Reddy VS. Evaluation of upper airway obstruction in infants with Pierre Robin sequence and the role of polysomnography - Review of current evidence. Paediatr Respir Rev. 2016;17:80-87. doi:10.1016/j.prrv.2015.10.001

7. Tan H-L, Kheirandish-Gozal L, Abel F, Gozal D. Craniofacial syndromes and sleep-related breathing disorders. Sleep Med Rev. 2015;27:74-88. doi:10.1016/j.smrv.2015.05.010

8. Leboulanger N, Fauroux B. Non-invasive positive-pressure ventilation in children in otolaryngology. Eur Ann Otorhinolaryngol Head Neck Dis. 2013;130(2):73-77. doi:10.1016/j.anorl.2012.06.001

9. Cielo CM, Marcus CL. Obstructive sleep apnoea in children with craniofacial syndromes. Paediatr Respir Rev. 2015;16(3):189-196. doi:10.1016/j.prrv.2014.11.003

10. Bonuck KA, Chervin RD, Cole TJ, et al. Prevalence and persistence of sleep disordered breathing symptoms in young children: a 6-year population-based cohort study. Sleep. 2011;34(7):875-884. doi:10.5665/SLEEP.1118

11. Smith CB, Walker K, Badawi N, Waters KA, MacLean JE. Impact of sleep and breathing in infancy on outcomes at three years of age for children with cleft lip and/or palate. Sleep. 2014;37(5):919-925. doi:10.5665/sleep.3660 
12. Cogswell JJ, Easton DM. Cor pulmonale in the Pierre Robin syndrome. Arch Dis Child. 1974;49(11):905-908.

13. Kaditis AG, Alonso Alvarez ML, Boudewyns A, et al. ERS statement on obstructive sleep disordered breathing in 1- to 23-month-old children. Eur Respir J. 2017;50(6).

doi:10.1183/13993003.00985-2017

14. Daniel M, Bailey S, Walker K, et al. Airway, feeding and growth in infants with Robin sequence and sleep apnoea. Int J Pediatr Otorhinolaryngol. 2013;77(4):499-503.

doi:10.1016/j.ijporl.2012.12.019

15. Abel F, Bajaj Y, Wyatt M, Wallis C. The successful use of the nasopharyngeal airway in Pierre Robin sequence: an 11-year experience. Arch Dis Child. 2012;97(4):331-334.

doi:10.1136/archdischild-2011-301134

16. Evans KN, Sie KC, Hopper RA, Glass RP, Hing AV, Cunningham ML. Robin sequence: from diagnosis to development of an effective management plan. Pediatrics. 2011;127(5):936-948. doi:10.1542/peds.2010-2615

17. Grigg-Damberger MM. The Visual Scoring of Sleep in Infants 0 to 2 Months of Age. J Clin Sleep Med JCSM Off Publ Am Acad Sleep Med. 2016;12(3):429-445. doi:10.5664/jcsm.5600

18. Grigg-Damberger M, Gozal D, Marcus CL, et al. The visual scoring of sleep and arousal in infants and children. J Clin Sleep Med JCSM Off Publ Am Acad Sleep Med. 2007;3(2):201-240.

19. Berry RB, Budhiraja R, Gottlieb DJ, et al. Rules for scoring respiratory events in sleep: update of the 2007 AASM Manual for the Scoring of Sleep and Associated Events. Deliberations of the Sleep Apnea Definitions Task Force of the American Academy of Sleep Medicine. J Clin Sleep Med JCSM Off Publ Am Acad Sleep Med. 2012;8(5):597-619. doi:10.5664/jcsm.2172

20. R Development Core Team. 2005. http://www.R-project.org.

21. MacLean JE, Fitzsimons D, Fitzgerald DA, Waters KA. The spectrum of sleep-disordered breathing symptoms and respiratory events in infants with cleft lip and/or palate. Arch Dis Child. 2012;97(12):1058-1063. doi:10.1136/archdischild-2012-302104

22. Montemitro E, Franco P, Scaillet S, et al. Maturation of spontaneous arousals in healthy infants. Sleep. 2008;31(1):47-54.

23. Schaefer RB, Stadler JA, Gosain AK. To distract or not to distract: an algorithm for airway management in isolated Pierre Robin sequence. Plast Reconstr Surg. 2004;113(4):1113-1125. 


\section{Legends}

Table 1: Patient characteristics before and during polysomnography (PSG). ${ }^{a}$ respiratory signs: snoring or noisy breathing or apneas or apparent life-threatening events or frequent arousals or respiratory effort or desaturation or stridor WA: weeks of amenorrhea, PSG : polysomnography, IQR: interquartile range

Table 2: Sleep characteristics in the prone and supine position. All data are expressed as median [IQR]. REM: rapid eye movement stage; NREM: non-rapid eye movement stages; TST: total sleep time

Figure 1: Effect of body position on obstructive apnea hypopnea index (OAHI) in 18 infants with Pierre Robin sequence. The thick line represents the threshold of obstructive events frequency under which infants would no longer require respiratory support (OAHI=10/hours of TST).

Table 3: Respiratory characteristics in prone and supine position. Data are expressed as median [IQR]. REM: rapid eye movement stage; NREM: non rapid eye movement stage; TST: total sleep time; $\mathrm{P}(\mathrm{tcCO}(2))$ : transcutaneous $\mathrm{CO}$. 
Table 1: Patient characteristics before and during polysomnography (PSG)

\begin{tabular}{lc}
\hline & $\begin{array}{c}\text { Study population } \\
\mathrm{n}=18\end{array}$ \\
\hline Sex ratio (M/F) & $9 / 9$ \\
Pierre Robin Sequence, $\mathrm{n}(\%)$ & $12(67)$ \\
Isolated PRS & $0(0)$ \\
Bone disease PRS & $6(33)$ \\
Associated or syndromic PRS & $3(17)$ \\
Antenatal diagnosis, $\mathrm{n}(\%)$ & $39[38-40]$ \\
Median gestational age, WA [IQR] & $44[27-70]$ \\
Median age at the PSG, days [IQR] & $4[3-4]$ \\
Median weight at the PSG, kg [IQR] & \\
Respiratory characteristics & $12(67)$ \\
Respiratory signs, $\mathrm{n}(\%)^{\mathrm{a}}$ & \\
Respiratory treatment during PSG, $\mathrm{n}(\%)$ & $15(83)$ \\
None & $2(11)$ \\
Non-invasive ventilation & $1(6)$ \\
Nasopharyngeal tube & \\
Digestive characteristics & $16(89)$ \\
Oral disorders, $\mathrm{n}(\%)$ & \\
Gastroesophageal reflux treatments, $\mathrm{n}(\%)$ & $14(78)$ \\
Thickened milk + proton inhibitor or anti acid & $2(11)$ \\
Thickened milk alone & $2(11)$ \\
Missing data & $11(61)$ \\
Nasogastric tube before PSG, $\mathrm{n}(\%)$ & $1(6)$ \\
Surgeries before PSG, $\mathrm{n}(\%)$ & $1(6)$ \\
Glossopexia & \\
Cleft closure & \\
\hline
\end{tabular}

${ }^{a}$ respiratory signs: snoring or noisy breathing or apneas or apparent life-threatening events or frequent arousals or respiratory effort or desaturation or stridor WA: weeks of amenorrhea, PSG : polysomnography, IQR: interquartile range 
Table 2: Sleep characteristics in the prone and supine position

\begin{tabular}{lccc}
\hline & Supine & Prone & p levels \\
\hline TST, minutes & $153[86-243]$ & $170[129-222]$ & 0.80 \\
Sleep efficacy, \% & $73[56-84]$ & $85[74-91]$ & 0.11 \\
Sleep efficiency, \% & $70[55-77]$ & $83[69-90]$ & 0.04 \\
Arousals index, n/h & $24[17-34]$ & $19[16-29]$ & 0.22 \\
Respiratory arousal index, $\mathrm{n} / \mathrm{h}$ & $19[11-28]$ & $15[6-25]$ & 0.06 \\
$\begin{array}{l}\text { Proportion of sleep stages } \\
\text { \% of total sleep time }\end{array}$ & & & \\
$\quad$ REM & $36[20-42]$ & $41[36-45]$ & 0.12 \\
$\quad$ NREM 1 & $19[12-29]$ & $15[11-20]$ & 0.03 \\
$\quad$ NREM 2 & $45[33-50]$ & $44[34-55]$ & 0.90
\end{tabular}

All data are expressed as median [IQR]. REM: rapid eye movement stage; NREM: non rapid eye movement stages; TST: total sleep time 


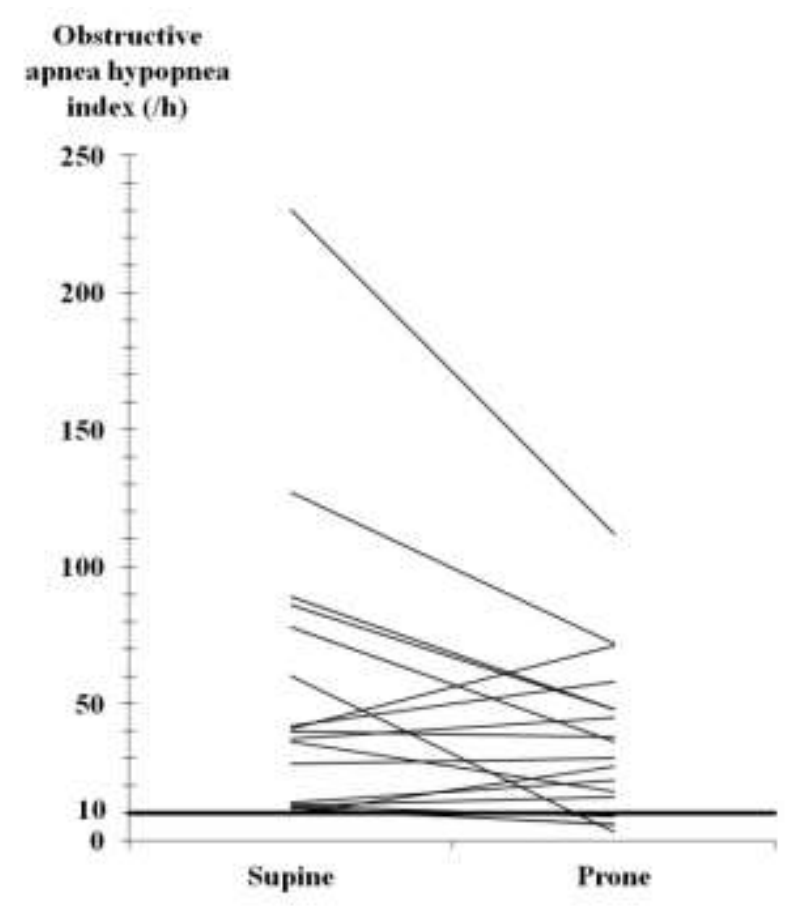

Figure 1: Effect of body position on obstructive apnea hypopnea index (OAHI) of total sleep time (TST) in 18 infants with Pierre Robin sequence. The thick line represents the threshold of obstructive events frequency under which infants would no longer require respiratory support (OAHI=10/hours of TST). 
Table 3: Respiratory characteristics in prone and supine position

\begin{tabular}{|c|c|c|c|}
\hline & Supine & Prone & p levels \\
\hline \multicolumn{4}{|l|}{ Obstructive apnea-hypopnea index (OAHI), n/h } \\
\hline TST & $39[13-74]$ & $33[17-48]$ & 0.13 \\
\hline NREM & $30[9-40]$ & $15[7-30]$ & 0.10 \\
\hline REM & $61[40-103]$ & $50[28-82]$ & 0.05 \\
\hline \multicolumn{4}{|l|}{ Obstructive apnea index (OAI), n/h } \\
\hline TST & $21[9-34]$ & 9 [4-34] & 0.06 \\
\hline NREM & $11[3-24]$ & $4[2-15]$ & 0.20 \\
\hline REM & $31[16-62]$ & $15[4-54]$ & 0.09 \\
\hline \multicolumn{4}{|l|}{ Obstructive hypopnea index (OHI), n/h } \\
\hline TST & $6[3-22]$ & $7[1-13]$ & 0.60 \\
\hline NREM & $3[1-15]$ & $3[1-9]$ & 0.20 \\
\hline REM & $8[2-28]$ & 15 [3-24] & 0.34 \\
\hline \multicolumn{4}{|l|}{ Central apnea index (CAI), n/h } \\
\hline TST & $3[1-6]$ & $3[0-4]$ & 0.26 \\
\hline NREM & $2[1-5]$ & $1[0-2]$ & 0.07 \\
\hline REM & $4[0-5]$ & $2[1-6]$ & 0.50 \\
\hline \multicolumn{4}{|l|}{ Mixed apnea index (MAI), n/h } \\
\hline TST & $3[1-11]$ & $3[1-5]$ & 0.08 \\
\hline NREM & $2[1-10]$ & $1[0-2]$ & 0.01 \\
\hline REM & $4[0-13]$ & 4 [2-9] & 0.50 \\
\hline Mean saturation, $\%$ & 97 [95-98] & 97 [94-97] & 0.78 \\
\hline \multicolumn{4}{|l|}{ Oxygen desaturation index (OD), n/h } \\
\hline$>3 \%$ & 34 [10-77] & $27[11-42]$ & 0.10 \\
\hline$>4 \%$ & $22[8-60]$ & $13[5-31]$ & 0.03 \\
\hline Mean $\mathrm{P}(\mathrm{tcCO}(2))$ levels, $\mathrm{mmHg}$ & $47[45-54]$ & 49 [43-55] & 0.32 \\
\hline Max $\mathrm{P}(\mathrm{tcCO}(2))$ levels, $\mathrm{mmHg}$ & $53[49-58]$ & $53[50-60]$ & 0.54 \\
\hline $\begin{array}{l}\text { Time spent with } \mathrm{P}(\mathrm{tcCO}(2)) \text { values }>50 \mathrm{mmHg} \text {, } \\
\text { \% TST }\end{array}$ & $29[0-100]$ & $38[0-93]$ & 0.9 \\
\hline
\end{tabular}

Data are expressed as median [IQR]. REM: rapid eye movement stage; NREM: non rapid eye movement stage; TST: total sleep time; $\mathrm{P}(\mathrm{tcCO}(2))$ : transcutaneous CO2. 